\title{
Sleep Musicalization: Automatic Music Composition from Sleep Measurements
}

\section{Tulilaulu, Aurora}

Springer-Verlag

2012-10-25

Tulilaulu , A , Paalasmaa , J , Waris , M \& Toivonen , H 2012 , Sleep Musicalization:

Automatic Music Composition from Sleep Measurements . in J Hollmén, F Klawonn \& A

Tucker (eds), Advances in Intelligent Data Analysis XI : 11th International Symposium, IDA 2012, Helsinki, Finland, October 25-27, 2012. Proceedings . Lecture Notes in Computer

Science, vol. 7619 , Springer-Verlag , pp. 392-403, International Symposium on Intelligent

Data Analysis (IDA) , Helsinki , Finland , 25/10/2012 . https://doi.org/10.1007/978-3-642-34156-4_36

http://hdl.handle.net/10138/37534

https://doi.org/10.1007/978-3-642-34156-4_36

submittedVersion

Downloaded from Helda, University of Helsinki institutional repository.

This is an electronic reprint of the original article.

This reprint may differ from the original in pagination and typographic detail.

Please cite the original version. 


\title{
Sleep Musicalization: Automatic Music Composition from Sleep Measurements
}

\author{
Aurora Tulilaulu ${ }^{1}$, Joonas Paalasmaa ${ }^{1,2}$, Mikko Waris $^{2}$, and Hannu Toivonen ${ }^{1}$ \\ 1 Department of Computer Science and HIIT, University of Helsinki, Finland \\ tulilaulu@gmail.com, hannu.toivonen@cs.helsinki.fi \\ 2 Beddit.com Ltd, Espoo, Finland \\ joonas.paalasmaa@beddit.com, mikko.waris@beddit.com
}

\begin{abstract}
We introduce data musicalization as a novel approach to aid analysis and understanding of sleep measurement data. Data musicalization is the process of automatically composing novel music, with given data used to guide the process. We present Sleep Musicalization, a methodology that reads a signal from state-of-the-art mattress sensor, uses highly non-trivial data analysis methods to measure sleep from the signal, and then composes music from the measurements. As a result, Sleep Musicalization produces music that reflects the user's sleep during a night and complements visualizations of sleep measurements. The ultimate goal is to help users improve their sleep and well-being. For practical use and later evaluation of the methodology, we have built a public web service at http://sleepmusicalization.net for users of the sleep sensors.
\end{abstract}

\section{Introduction}

Understanding data is a central goal of statistics and data mining. Numerical or textual representations can convey very exact results of data analysis, but they are not always the most convenient form for the consumer of the results. Different charts, and data visualization in more general, have proven to be a powerful way of illustrating general properties, distributions, trends and patterns in data, even if details may be missed. But how to represent data analysis results so that they give the user a deeper feeling of the data, perhaps even an emotional one?

In this paper, we consider sleep analysis as a data analysis problem, and propose a novel data analytical approach for it: data musicalization. We define data musicalization as the process of automatically composing music from given data, with the goal of perceptualizing it. Figuratively speaking, the data is used to inspire a composition algorithm that produces a novel piece of music. This is in contrast to classical data sonification where data is just mapped to sounds with little if any intention to generate music, and typically with no control at all over the musicality of the result.

Measuring and tracking one's sleep over extended periods of time can help improve sleep and well-being. Sleep analysis methods were previously developed 
for clinical use to help patients with sleep disorders. State-of-the-art sensor technology now allows unobtrusive sleep analysis at home, with two implications for data analysis. First, deriving high-level sleep data, e.g., sleep stages from the physiological sensor data is a complex data analysis task. Second, the users are ordinary people who want to get a more analytical feeling or their sleep, not sleep doctors specialized in reading hypnograms (charts of sleep stages) and actigrams (charts of movements).

The main contribution of this paper is to propose data musicalization as a tool to aid data analysis and understanding. The proposed methodology, called Sleep Musicalization, consists of non-trivial data analysis methods for sleep analysis on one hand, and of automatic composition of music from the sleep analysis results on the other hand. For instrumentation, we use a modern, commercially available mattress sensor ${ }^{3}$ that can be used to detect sleep stages as well as respiration, heart rate and movements. We have described these sleep analysis methods in detail elsewhere $[1,2]$ and in this paper we just give a brief overview of them.

Based on the sleep information, a piece of music is automatically composed. The goal is that the music reflects the structure of sleep during the night and gives the user a feeling of her sleep. We give stochastic composition algorithms to produce the harmony, melodies, and rhythm of music, based on musicological principles. Their novelty is not as much in the quality of the music per se as in making the composition reflect the given attributes of sleep.

We have set up a web service, http://sleepmusicalization.net, that supports end-to-end sleep analysis and musicalization. This service also allows us to test the approach with real end users. Some example compositions shared by the users of the service are publicly available.

This paper is structured as follows. We start by reviewing background and related work in Section 2. The methods we use for both sleep data analysis and automatic composition are described in Section 3. We discuss the methods and initial results in Section 4. Section 5 contains concluding remarks. An appendix contains informal definitions of some musical terms.

\section{Background and Related Work}

\subsection{Sleep Measurement}

The aim of medical sleep measurement is to divide sleep into sleep stages and diagnose various sleep disorders. The standard method for measuring sleep stages for medical purposes is polysomnography, which involves attaching biopotential electrodes to the head. Based on the measured signals, sleep can be divided into five sleep stages: wakefulness, REM (rapid eye movement) sleep and three categories of non-REM sleep: N1, N2 and N3 [3]. Stages N1 and N2 are called light sleep and N3 deep sleep. The classification into sleep stages, also known as scoring, is normally done manually by a trained sleep technician. Additional

\footnotetext{
${ }^{3}$ http://www.beddit.com
} 
respiration sensors are typically used for diagnosing sleep-related breathing disorders. Polysomnography costs hundreds of euros per measurement night and is not commonly available.

The other widely used medical sleep measurement method is actigraphy, where the patient wears a wrist accelerometer typically for one week, 24 hours a day. Actigraphy measurement is convenient for the patient, but has limited diagnostic capability. It can assist in determining sleep patterns in healthy adult populations and diagnosing circadian sleep-wake disorders [4].

There also are non-medical sleep measurement products for self-help use. Zeo Sleep Manager measures electroencephalography (EEG) as well as actigraphy with a wireless sensor on the forehead, and classifies sleep into wakefulness, REM sleep, light sleep and deep sleep. The device has been on the market for several years and has validated accuracy [5]. Fitbit, BodyMedia FIT, Jawbone UP, SleepTracker, LARK and WakeMate are wrist actigraphy devices for sleepwake classification. Classifying sleep periods into REM, light and deep varieties is not performed, because the wrist measurement provides insufficient information.

The sensor that we use for sleep measurement, Beddit, differs from other selfhelp devices in that the measurement is fully unobtrusive: the data is acquired with a thin force sensor placed under the mattress. Other devices require wearing a sensor in the wrist or on the forehead, which may deteriorate sleep quality.

With most of the self-help devices, sleep information is presented visually to the user in a smartphone interface and a web service. We are not aware of other attempts to produce music from sleep data.

\subsection{Music Generation from Physiological Signals}

Sonification, i.e., the use of sounds to convey information is a wide area of research [6]. We here focus on methods that sonify physiological signals (such as the ones used to measure sleep).

Research on generating music from physiological measurements mostly deals with EEG and electrocardiography (ECG). The first device to generate music from physiological measurements was the "encephalophone", from the 1940s [7]. It generates audio from measured brain waves for both medical and musical purposes. Various artists have since made similar instruments. For example, Krzysztof Penderecki's Polymorphia (1963) was based on encephalographic pitch notation derived from EEG data.

The first commercial biomusic production environment, BioMuse, was introduced in late 1980s [8]. Notable contemporary performances include, to name a few: underwater-EEG-ECG DECONcert [9], Multimodal Brain Orchestra [10] as well as Baroque Duet for Cello, Violin, 2 Hearts (ECG) and 2 Minds (EEG) by Barrass and Whitmer, performed at the Sound and Music Computing Conference in 2010. 


\subsection{Automatic Composition of Music}

Algorithmic composition of music is a much older phenomenon than computers, and many kinds of formal processes have been proposed to generate music. Already around year 1026, Guido d'Arezzo introduced a technique for generating melodies to accompany a text [11]. One of the most famous examples of automatic composition is Mozart's Musikalisches Würfelspiel, a dice game for generating waltzes. Prior to computers, some mechanical composition machinery also existed [12].

The first computer composition, The Illiac Suite for String Quartet, was generated by a program by Hiller and Isaacson in 1956. Current state-of-theart includes David Cope's program called Emily Howell who composes complex music in her own style [13].

Mapping data to sounds or music is an old idea, too. Several attempts have been made in biology. For instance, Protein music was obtained by translating coding sequences into sound [14]. A contemporary example with CDs on sale is Your DNA Song ${ }^{4}$, a service for turning DNA into music by mapping the 22 amino acids to 22 different notes and then playing out the result with 3000 nucleotides per minute. These systems sonify data essentially by translating it to sound. In contrast, we are interested in having music composed automatically, with the data given just for guidance. This way several musical characteristics are under the control of the composition algorithm, not just the data.

Music can be composed using many different approaches and their combinations. Typical examples include cellular automata, fractals, grammars, constraints, pattern matching, and many different stochastic processes, like Markov chains and different uses of distributions. A review of these approaches is outside the scope of this paper, but see, e.g, [12] for an overview. We use relatively simple Markov chains and random walks, and the novelty is in making the music reflect sleep.

Approaches to automated composition can also be classified by their interactivity with the user. Some systems generate accompaniments for the user's music, or generate short parts of melody [12]. Some systems also accept feedback from the user [12]. One of the better known examples of this is the band in a box software that generates accompaniments with the chosen chords in a style the user prefers. Other systems, like the one to be described in this paper, do the whole composition process autonomically.

\section{Methods}

We next present the Sleep Musicalization methodology in three parts. First, we describe the data analysis methods we use for sleep measurement. Since these methods have been reported elsewhere, we here just give an overview of them. Second, we present the composition algorithm that produces music from sleep

\footnotetext{
${ }^{4}$ http://www.yourdnasong.com/
} 
measurements. Third, we describe the implementation of Sleep Musicalization as a web service.

The Python source codes of the musicalization ${ }^{5}$ and web service ${ }^{6}$ components are available for download.

\subsection{Sleep Measurement}

Sensor Data Collection The raw signal is acquired using a thin, flexible piezoelectric force sensor by Beddit, measuring 70 by $4 \mathrm{~cm}$, placed under the mattress topper or mattress. The signal from the sensor is sampled at $140 \mathrm{~Hz}$. An excerpt of the measured signal is shown in Figure 1.

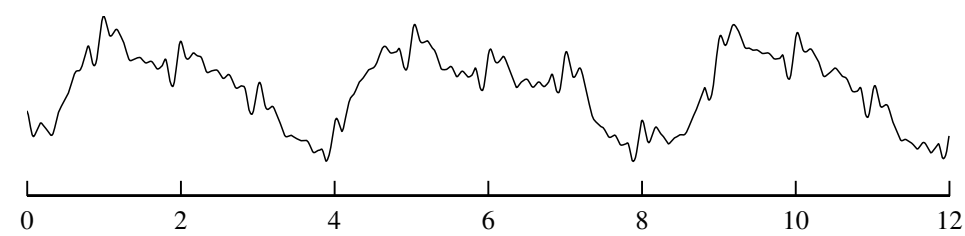

Fig. 1. A 12-second signal excerpt from the Beddit sensor. Respiration is the cyclic lowfrequency waveform with 4-second period. The heartbeats occur around every second.

A tailor-made miniature Linux computer receives the signal, performs analogto-digital conversion, and sends the signal to a web service for analysis. Around 300 kilobytes of compressed data is sent per a measured hour of sleep. In addition to the force signal, information related to the sleeping environment is measured every minute: temperature, noise level and brightness.

Data Analysis for Physiological Parameters The force signal contains heartbeats as well as respiratory and movement activity. In Figure 1, the overall trend corresponds to respiration cycles, while heartbeats can be detected from the finergrained fluctuations of the force signal. Sleep analysis greatly depends on heart rate variability, and this can be obtained from the signal in the form of inter-beat intervals. The measurement of heart rate from a force signal is called ballistocardiography, and it is an established technique [15]. We have shown elsewhere that heart rate intervals can be detected accurately from our current sensor data [2]. The low-frequency phenomenon of the force signal is also analyzed [1], in order to detect the length of each respiration cycle and variations in them. This information is also needed in the sleep analysis.

Sleeper movements are detected by analyzing individual abrupt changes in the force signal. The resolution of movement detection is set at three seconds, i.e., a movement is recorded to occur at most every three seconds.

${ }^{5}$ https://github.com/Tulilaulu/Sleep-musicalization

${ }^{6}$ https://github.com/beddit/sleep-musicalization-web 
Data Analysis for Sleep Stages Now, based on heart rate and respiration variability as well as movement information, the time spent in bed is segmented into periods of wakefulness, REM sleep, light sleep and deep sleep.

The classifier is based on a generative model of heart rate, respiration and movement features given sleep stage. Transitions between sleep stages are described with Markov models. On a high level, the generative model works as follows. The levels of heart rate and respiratory variation are highest during wakefulness and REM, slightly lower in light sleep, and lowest in deep sleep. Wakefulness has a high level of movement activity, REM and light sleep stages permit some movements, while in deep sleep the level of activity is very low.

A full validation of the classifier is in progress; the details of the classifier and its validation will be published elsewhere.

The result of sleep analysis thus consists of the structure of the sleep (hypnogram: segments of different sleep stages) as well as information about the heartrate and movements of the sleeper during the night. Figure 2 is a screenshot from the Beddit service illustrating what the analysis results look like when visualized.

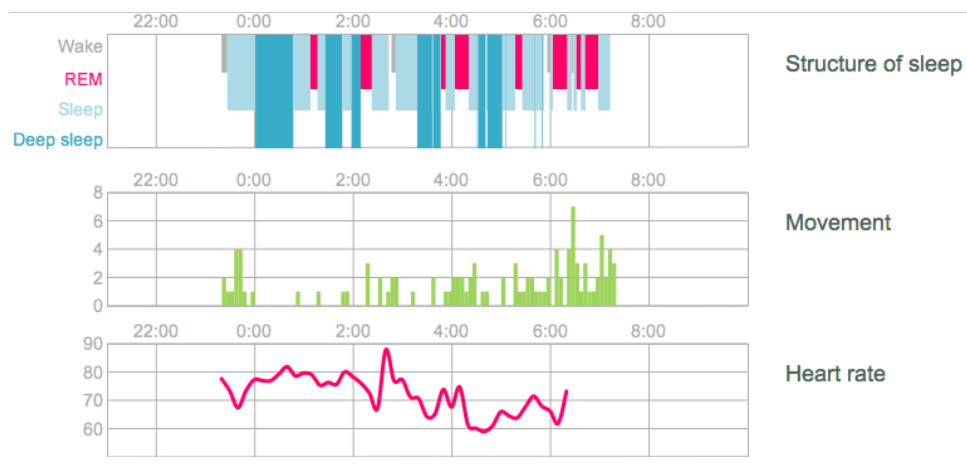

Fig. 2. The key sleep information for one night.

\subsection{Automatic Composition of Music}

In Sleep Musicalization, a novel piece of music is automatically composed from the sleep measurements of one night at a time. We currently use the following information: sleep stages, heart rate, and movements. The composition method has been designed to make these aspects clearly audible in the music, as will be described below. The piece is roughly compressed to ratio 1:120, i.e., one second of music corresponds to two minutes of sleep or, in other words, eight hours of sleep results in a song of about 4 minutes.

Preparations Before the actual composition starts, sleep stages that are very short are removed to give more stability to the music. 
A scale ${ }^{7}$ is chosen at random by picking the starting point of the scale and its type, a major scale or a harmonic minor scale, the most common Western scales of music. The default is major, however pieces in minor can be generated if the user so wishes.

The method next randomly decides if the bars will have 3 or 4 beats. The length of a bar is significant musically; the method also processes music one bar at a time and associates one chord with each bar.

Generation of a Chord Sequence The sequence of chords is first generated with a Markov chain. It is hand-coded to have meaningful transition probabilities between chords. (Note that the Markov chains used to generate chords or other musical aspects below have nothing to do with the Markov models of sleep stages used in sleep analysis.)

For simplicity, the Markov model has maximum degree 2, i.e., it only takes into account two previous chords when generating the next one. Not all pairs of two last chords have a transition distribution assigned to them and in those cases the next chord is chosen using only the previous chord. At the moment we only use chords that consist of exactly three notes that are in the chosen scale. (In music in general, chords are often used that are not native to the key. We only use 7 elementary chords per piece to cut back on complexity.)

The probabilities of the chords have in general been chosen to reflect their frequencies and dominance in music using the given key. Exceptions to this rule are transition probabilities in the Markov chain that make sure that chords that need to be resolved will be resolved (i.e., from the dissonant seventh chord of the key, it is best to move to the first chord). Once the sequence of chords has been generated for the piece of music, the method moves on to compose a melody.

Melody Generation Intervals can be classified into steps and skips. Major and minor seconds and unison are steps. Skips, in turn, are any larger intervals of up to an octave up or down. This division to steps and skips is useful because small intervals appear more commonly in music than big ones.

The intervals that form the melody are generated using another Markov chain. The Markov chains are constructed so that the total probability of steps is roughly equal to the total probability of skips. Additionally, the probabilities of transitions between some specific intervals has been set. If the last interval was a skip upward, the next is either a step or a skip downward and if it was a skip downward, it is followed by a skip upward or a step. If the resulting note is not in the scale or is in bad dissonance with the current chord it is rejected, and the Markov chain is used to generate a new interval instead.

In order to have some internal coherence in the music, the melody generation procedure uses musical themes. Different sleep stages have a different theme each, so they will have more clear identities in the music. (As a special case, the state of being absent from bed generates silence.) As the very first step of melody generation, a theme of two bars is generated for each sleep stage for later use.

\footnotetext{
${ }^{7}$ See Appendix for a glossary of some musical terms.
} 
The actual melody is composed for one sleep stage segment at a time. The melody for a segment starts with the theme of the sleep stage, after which the rest of the segment is filled with the melody generation method.

Generation of Rhythm Note lengths are generated with a Markov chain of degree 1, but the degree will be increased later to get more better results. The values are chosen so that they favour multiples of the same length as it is common for especially shorter note lengths to appear in groups. They also favour rhythmic patterns that match the beats. For instance, if the last note is 1.5 beats long, the next one is likely to be 0.5 . However, if the remaining length of the segment at hand is less than 2.5 beats, then the note will last until the end of the segment. This avoids the practical problem of going over the segment length, but it is also a good way to give the last note of the segment a bigger probability of being longer. The rhythm also slightly varies with respiration. Higher respiration rate make short rhythms sligthly more likely and slower respiration rate longer rhythms a bit more likely.

Generation of Accompaniment The accompaniment is also generated sleep stage segment by segment. It also changes according to the sleep stage: for each stage, there is a different generating function. The awake accompaniment just consists of short notes on the base of the chord. The one for REM sleep consists of quite a fast pattern using all the notes of the chord. The accompaniment of light sleep is a generic up-and-down pattern of the notes of the chord, slightly slower than in REM sleep. Finally, the deep sleep accompaniment consists of long notes in a slow pattern. All of the generators use only notes of the current chord. In the accompaniment, slight emphasis is added on the first beat or all beats, depending on sleep stage.

Volume to Reflect Sleeper Movements The actigram data (movement information) is preprocessed so that it contains information on the density of the movements for each beat or music. If there are a lot of movements, the volume rises and if there are very little movements, the volume goes down. (In addition, there can be other changes to volume, such as emphasis on the beats.)

Regulation of Tempo The tempo (beats per minute) varies during the music, reflecting the heart rate. The heart rate is transformed to make changes more pronounced. First, it is approximated in a piece-wise constant manner so that tempo changes are discrete. This is implemented by taking a cumulative moving average and comparing it to the current constant approximation. If the absolute difference exceeds a pre-determined threshold, then the current value is taken as the next constant approximation. Second, the starting times of each point in the averages and the tempos attached to them are processed so that slower tempos get less beats and faster tempos get more beats to keep the song moving through the sleep stages at a constant pace. Then, the relative magnitude of changes is amplified. The heart rate estimate is multiplied by four and 180 is subtracted form the result. Another interesting option for regulation of tempo would be to use respiration rate, which is also available from the sleep analysis. 
Sound Synthesis We use Kunquat ${ }^{8}$ for the actual sound generation, given the composed music. Kunquat uses an event-based approach to represent music. There are different events for starting a note, stopping a note, changing the tempo and changing the force of the note. The pitch of a note is represented as a number where 0 is equal to $440 \mathrm{~Hz}$ and an addition or subtraction of 100 moves the pitch a minor second. The program also does not deal with time as seconds, but uses beats as its main time unit. Our implementation uses these units internally. In Kunquat, music is represented in tracks, where only one note can be playing at a time. For this reason, the melody and accompaniment are generated to their own tracks. After the composition of music is ready, it is given to Kunquat which produces an audio file.

\subsection{Implementation as a Web Service}

We have implemented a web service (http://sleepmusicalization.net) where sleep music can be generated and accessed conveniently by users of the Beddit device. This is a valuable tool also for research, as it will allow end user studies of the Sleep Musicalization methodology and the music generated. At the web site, others interested can listen to music published by Beddit users.

In the end-to-end system, a user of the Beddit sleep tracking service first grants the sleepmusicalization service access to her data (Figure 3). This authentication is implemented using OAuth2 protocol. When the user wants to listen to her sleep as music, she initiates the composition process at either the sleepmusicalization or Beddit site. The sleepmusicalization service then retrieves sleep measurement data from the Beddit service and runs the music composing algorithm. The generated music can then be listened to on a web page, or published for others to listen and share in social media.

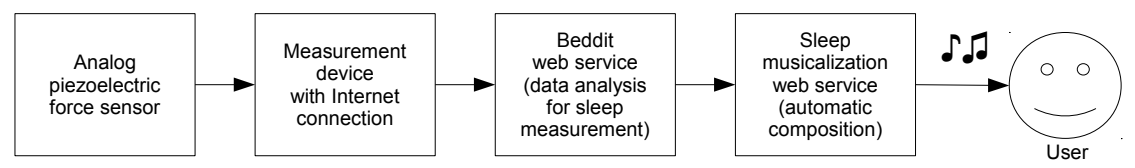

Fig. 3. The end-to-end flow of sleep information, from an analog force signal to sleep measurements to automatically composed music.

\section{Results and Discussion}

The goal of Sleep Musicalization is to provide a novel way to perceptualize sleep measurements, complementing their visualizations. The Sleep Musicalization method as described above has been implemented and can be freely used

\footnotetext{
${ }^{8}$ http://kunquat.org
} 
at http://sleepmusicalization.net, where example results are also available for listening.

At this phase of the research, it is too early to draw conclusions about the value and success of the methodology. Our own, subjective opinion is that the key characteristics of sleep, especially the sleep stages, are already perceptualized well with the current method. An objective evaluation and validation is yet to be carried out.

The quality of the music could be improved a lot. The current composition algorithms are relatively simple and, as a result, the musicality of the melodies is still questionable. A large body of literature on composition algorithms exist (see, e.g., [12]) and could be used to implement better methods. It is likely that mixing other methods with the ones used now will result in better compositions. One possibility could also be to program the system to learn from feedback or other compositions, as has been implemented elsewhere [13].

There currently are some practical limitations which could in principle be easily circumvented. For instance, we only use three tracks, i.e., at most three notes are playing at the same time. For the quality of the result this is not a crucial point, however. Many of the choices we have made in the music composition have viable alternatives, like adding other scale types, allowing a bar to have some other number of beats than 3 or 4 , or accepting notes that are not in the scale.

The current set of sleep measurements contains the most important characteristics of sleep, but additional data would also be available. The most relevant additional physiological measurements are heart rate variation and respiration rate variation. We plan to incorporate such information, e.g., in the average pitch or volume of the music, or in the frequency of the rhythm.

The Beddit device, of which the force sensor is just one part, also provides information about the environment. Ambient levels of light, noise, and temperature could be useful additions, as possible explanations of some sleep events.

From a data mining and learning perspective, a fascinating challenge would be to learn a musical style from a given corpus of musical scores. Given a set of songs, say, of Beatles, the system should learn to produce new music in the style of Beatles, and be able to adjust it according to sleep measurements. This would allow, in principle, the user to select the style of music in which her sleep is musicalized.

\section{Conclusion}

We have proposed data musicalization as a novel way to represent data or data analysis results to the user. We presented Sleep Musicalization, a methodology combining state-of-the-art sleep measurement sensors and methods with automated composition algorithms. Sleep Musicalization produces music that reflects the user's sleep during a night, with the aim of complementing existing visualization methods for sleep measurements. The ultimate goal is to help users understand and track their sleep, in order to improve their sleep and well-being. We 
hope that musicalization of sleep measurements can make this tracking process more attractive and fun for some users.

Data musicalization is based on composing music, not just mapping data to sounds as is usual in sonification. In this paper, we presented stochastic composition algorithms that are constructed specifically for sleep measurements.

The next phase in this work will be validation of the Sleep Musicalization approach. How much and what kind of information about sleep is actually conveyed to the users? Does it provide some additional value to the users, complementing current graphical results? The web service will allow us to gather actual user experiences and feedback. Other future work will include improving composition methods.

The sleepmusicalization.net service has now been designed for the Beddit sensor. It should be conceptually relatively easy to adapt the composition algorithm to work with sleep data from sources other than Beddit, e.g., Zeo Sleep Manager.

An interesting research question is if the data musicalization approach is applicable to other data sets and application areas. In the current composition algorithm, we already perceptualize discrete state variables (sleep stages), continuous variables (heart and respiration rates), as well as event data (sleeper movements). Could similar ideas and methods be applied to perceptualize other temporal data sets?

Acknowledgments We would like to thank Tomi Jylhä-Ollila for help with the Kunquat system.

This work has been supported by the Algorithmic Data Analysis (Algodan) Centre of Excellence of the Academy of Finland (Grant 118653).

\section{References}

1. Paalasmaa, J., Leppakorpi, L., Partinen, M.: Quantifying respiratory variation with force sensor measurements. In: 33rd Annual International Conference of the IEEE Engineering in Medicine and Biology Society, EMBC'11. (2011) 3812-3815

2. Paalasmaa, J., Waris, M., Toivonen, H., Leppakorpi, L., Partinen, M.: Online monitoring of sleep at home. In: 34th Annual International Conference of the IEEE Engineering in Medicine and Biology Society, EMBC'12. (2012)

3. Iber, C., Ancoli-Israel, S., Chesson, A., Quan, S.F.: The AASM manual for the scoring of sleep and associated events: rules, terminology and technical specifications. American Academy of Sleep Medicine (2007)

4. Morgenthaler, T., Alessi, C., Friedman, L., Owens, J., Kapur, V., Boehlecke, B., Brown, T., Chesson Jr., A., Coleman, J., Lee-Chiong, T., Pancer, J., Swick, T.: Practice parameters for the use of actigraphy in the assessment of sleep and sleep disorders: An update for 2007. Sleep 30(4) (2007) 519-529

5. Shambroom, J.R., Fabregas, S.E., Johnstone, J.: Validation of an automated wireless system to monitor sleep in healthy adults. Journal of Sleep Research 21(2) (2012) 221-230 
6. Kramer, G., Walker, B.N.: Sound science: Marking ten international conferences on auditory display. ACM Transactions on Applied Perception (TAP) 2(4) (2005) 383-388

7. Henry, T.K.: Invention locates hurt brain cells. New York Times (March 2, 1943) 21

8. Knapp, R.B., Lusted, H.S.: A bioelectric controller for computer music applications. Computer Music Journal 14(1) (1990) 42-47

9. Mann, S., Fung, J., Garten, A.: DECONcert: bathing in the light, sound, and waters of the musical brainbaths. In: ICMC 2007: International Computer Music Conference. (2007)

10. Le Groux, S., Manzolli, J., Verschure, P.F.M.J.: Disembodied and collaborative musical interaction in the multimodal brain orchestra. In: NIME'10: Proceedings of the International Conference on New Interfaces for Musical Expression. (2010)

11. Kirchmeyer, H.: On the historical constitution of a rationalistic music. Die Reihe 8 (1968) 11-24

12. Roads, C.: The computer music tutorial. The MIT Press (1996)

13. Muscutt, K.: Composing with algorithms: An interview with David Cope. Computer Music Journal 31(3) (2007) 10-22

14. King, R.D., Angus, C.G.: PM - protein music. Bioinformatics 12(3) (1996) 251252

15. Pinheiro, E., Postolache, O., Girao, P.: Theory and developments in an unobtrusive cardiovascular system representation: Ballistocardiography. Open Biomedical Engineering Journal 4 (2010) 201-216

\section{Appendix: Glossary of some musical terms}

scale a series of notes differing in pitch according to a specific scheme; major scale and harmonic minor scale are typical Western scales

key a major or minor scale

minor second (or semitone) the smallest interval in use in Western music, e.g., the distance between two closest keys in a piano

major second (or whole tone) an interval as big as two semitones

octave an interval as big as 12 semitones, the distance between two notes of the same name (for example $\mathrm{c}$ to $\mathrm{c}$ ).

chord a combination of a minimum of three notes; the chords in this paper are made by taking a base note for the chord and adding the notes that are 2 up and 4 up from it in the given scale 